\title{
ANÁLISE DE RISCO DE QUEDA DE ÁRVORES: Tilia tomentosa Moench
}

\author{
TREE RISK ASSESSMENT: Tilia tomentosa Moench \\ Ana Luísa Pereira de Carvalho', Danielly Ferreira², Maria Cristina Morais Penas Nabais dos Santos ${ }^{3}$
}

\section{RESUMO}

Lidar com os riscos inerentes ao processo de arborização nas cidades é o grande desafio para a gestão do espaço urbano. Com o objetivo de analisar o risco de queda de árvores, foi desenvolvido e aplicado formulário de inspeção visual. Foram realizadas tomografias acústicas como ferramenta complementar de avaliação da condição mecânica da estrutura do tronco de árvores. A coleta de dados é relativa a exemplares vivos da espécie Tilia tomentosa Moench, no Parque Dr. Manuel Braga, em Coimbra/Portugal. Os resultados da inspeção visual subsidiaram a seleção dos exemplares submetidos à tomografia acústica e culminaram na classificação das árvores em termos de risco, sugerindo a necessidade de monitoramento dos ramos das árvores. Os resultados obtidos a partir das tomografias acústicas são coincidentes com estruturas consideradas mecanicamente estáveis, mesmo para aquelas visualmente consideradas condenadas. A inexistência de um banco de dados mundial sobre árvores urbanas e as dificuldades inerentes ao diagnóstico contribuem para aumentar a complexidade da análise de risco de queda de árvores, acentuando a necessidade de viabilizar suporte técnico-científico, para garantir prevenção de danos e adequada tomada de decisão quanto à realização de intervenções nas árvores do ambiente urbano. Espera-se com o presente contribuir para estudos de Tilias.

Palavras-chave: Parque urbano; Tomografia de árvore; Avaliação do lenho.

\section{ABSTRACT}

Dealing with risks inherited to afforestation process in cities is a major challenge for urban management. In order to analyze the risk of tree fall, a visual inspection form was developed and applied. Acoustic tomography was performed as a complementary tool to evaluate the mechanical condition of the tree trunk. Present data is related to live specimens of Tilia tomentosa Moench, at Dr. Manuel Braga Park, in Coimbra / Portugal. The results of the visual inspection supported the selection of specimens submitted to acoustic tomography and culminated in the classification of the trees in terms of risk, suggesting the need for monitoring the tree branches. The results obtained from acoustic tomography are coincident with structures considered mechanically stable, even for those visually considered condemned. The lack of a worldwide database on urban trees and the difficulties inherited to the diagnosis contribute to the complexity of the analysis of the risk of tree fall. It's necessary to provide technical and scientific support to ensure damage prevention and decision making about urban trees. It is expected with the present study to contribute to studies of Tilia.

Keywords: Urban park; Tree tomography; Wood evaluation

Recebido em 04.06.2019 e aceito em 06.09.2019

1 Bióloga. Mestre em Biologia Bolsista da Universidade de Coimbra. Coimbra/Portugal. Email: alp.carvalho1@gmail.com

2 Engenheira Florestal. Mestre em Biodiversidade e Biotecnologia Vegetal. Analista Ambiental em Instituto do Meio Ambiente e dos Recursos Hídricos do Distrito Federal. Brasília/DF. Email: danyflorestal@gmail.com

3 Bióloga. Doutora em Ecologia. Professora auxiliar na Faculdade de Ciências e Tecnologia na Universidade de Coimbra. Coimbra/Portugal. Email: crnabais@bot.uc.pt 


\section{INTRODUÇÃO}

A gestão de uma área urbana em expansão é um desafio. Por vezes, nesse processo, as árvores e a vocação natural do local acabam por ser negligenciadas no momento da tomada de decisão relativa ao ordenamento urbano. Como consequência da fragmentação e da perda de áreas verdes dentro das zonas urbanas, perdem-se benefícios inerentes à manutenção das árvores nas áreas urbanas, como o controle de condições naturais adversas e a capacidade de abrigar repositórios de biodiversidade (FERREIRA et al., 2013).

A árvore no meio urbano pode ser vista apenas pelo seu caráter paisagístico, não sendo objeto de atenção às funções de transporte de água e de reserva de nutrientes. Tais funções são primordialmente exercidas pelo alburno, que passa a ser convertido em cerne na medida em que o gasto energético para sua manutenção aumenta até limites próximos de 13\% da energia obtida por meio da fotossíntese. A indicação fisiológica de conversão de alburno em cerne é favorável à consolidação da árvore como estrutura na paisagem. Isso nos leva a perceber que existe uma associação entre os elementos paisagísticos e suas naturezas, na qualidade de matéria. No caso da árvore, como ser vivo, sua qualidade primordial é adaptar-se ao ambiente em que se encontra, muitas vezes levando centenas de anos para atingir níveis de adaptação que assegurem a permanência de sua espécie (RUXTON, 2014).

Certo é que nem sempre essa adaptação é condizente com o interesse puramente antropocêntrico. Os governos buscam evitar a responsabilização por danos causados pelas árvores e o impacto de eventuais processos judiciais, optando por remover as árvores dos passeios públicos, adotando um modelo de gestão de prevenção pura de riscos. Enquanto isso, a análise de risco de queda de árvores evoluiu como ciência e hoje é definida pela percepção de segurança, com o uso ou não de ferramentas, para definir níveis aceitáveis de risco, sendo que, na ausência destas, a política local, as emoções, a influência de líderes comunitários, o trabalho individual de funcionários e a atitude de moradores acabam por se sobrepor a critérios técnicos mais objetivos (BUCKERIDGE, 2015).

A arborização do Parque Dr. Manuel Braga acompanha o processo da cidade de Coimbra e a do país como um todo. Em Portugal, espécimes autóctones e espécies exóticas foram plantadas indiscriminadamente, sem quaisquer estudos de ecologia que possibilitassem prever minimamente o desenvolvimento das árvores como indivíduos, menos ainda sua integração na paisagem. Como consequência do plantio de árvores escolhidas por sua beleza e frondosidade, em detrimento de sua adaptabilidade ao meio, foram frequentes as intervenções de poda, atualmente não recomendadas em Coimbra, sabidamente pelo alto nível de infeções porque passaram as árvores da cidade. Alamedas inteiras de plátanos foram suprimidas devido ao cancro, ulmeiros abatidos devido à grafiose e outras doenças associadas 
à fragilização dos sistemas vegetais expostos às podas também foram registradas em consequência das atividades previstas nos calendários de poda municipais. Simplesmente podar ou abater árvores sem uma avaliação aprofundada tem se mostrado um grande equívoco na gestão urbana (PAIVA, 1996).

Justifica-se o presente trabalho pela necessidade de serem integrados às rotinas de gestão do espaço urbano os procedimentos sistemáticos de identificação, análise e avaliação do risco de queda de árvores, subsidiando a aplicação de políticas, que possibilitem mitigar, monitorar e gerenciar o risco de queda de árvores no ambiente urbano. A tomografia acústica ou por impulso, além de outras técnicas não destrutivas como os penetrógrafos, podem ser utilizadas como ferramentas, isolada ou cumulativamente, que possibilitam realizar análises de risco de queda de árvores que compõem a paisagem urbana. Essa avaliação, em conjunto com a inspeção visual, possibilita a realização de uma melhor análise de sua estrutura e de sua estabilidade, o que é essencial em se tratando da gestão desse tipo de recurso natural, esteja a árvore a integrar um grande povoamento florestal, uma floresta natural ou uma área urbanizada. Esse manejo terá impacto sobre a paisagem e sobre o uso adequado do solo.

A análise da estrutura da árvore perpassa o estudo das propriedades físicas e mecânicas do lenho, desde o sistema radicular e sua interação com o solo e tronco, a copa e as condições de crescimento, tanto as intrínsecas de cada espécie, como as relativas ao ambiente (SHORTLE; DUDZIK; SMITH, 2010).

Quanto aos movimentos mecânicos potencialmente comprometedores do sistema estrutural e da estabilidade da árvore citam-se os principais como sendo: a) o momento de rotação, devido a uma força lateral sobre o sistema raiz-tronco, capaz de produzir o efeito de uma alavanca, em face do tamanho da árvore e da sua capacidade de absorção de energia oriunda de uma carga, seja ela de origem antrópica ou natural; e, b) a resistência à flexão do tronco, devido à dificuldade de prever as reações da madeira após a absorção da energia exercida por uma carga que provoque a deformação do tronco. O fenómeno de interação gravitrópica no tronco da árvore pode ser modelado a partir da adaptação da teoria das vigas, já que em princípio o caule das árvores, como estrutura delgada, está sujeito aos efeitos de flambagem. Em biomecânica natural da árvore devem ser avaliados fatores como as variações no material ao longo do tempo, já que tanto o peso da estrutura se modifica continuamente, quanto as consequências advindas da maturação das células na madeira, decorrente da atividade cambial, são diferenciadas e imprevisíveis (ALMÉRAS; FOURNIER, 2009).

O estado mecânico resultante da ação simultânea de crescimento e de carga não pode ser previsto da mesma maneira que em uma estrutura isotrópica. $O$ efeito das cargas exercidas sobre um caule e a reação gravitrópica da árvore são, conjuntamente, os responsáveis diretos pelo equilíbrio da árvore como estrutura, em um ciclo de compensação de 
forças caracterizado por perturbação e compensação que depende essencialmente da eficiência da resposta gravitrópica. Portanto, o gravitropismo é o parâmetro crítico determinante do equilíbrio biomecânico. É certo que a sensibilidade das características biomecânicas envolvidas neste equilíbrio tem influencia sobre os modelos paramétricos desenvolvidos. Estudar os dados principais que afetam tal sensibilidade contribui para a calibração de um bom modelo em biomecânica (ALMÉRAS; FOURNIER, 2009).

Usualmente o efeito das variações de densidade na madeira, ao longo do sentido radial, é considerado no módulo de elasticidade homogeneizado da seção observada, e assume valor constante. No entanto, sabe-se que o mais comum é que a rigidez da madeira mude, conforme varia a idade do câmbio, sendo a madeira quanto mais jovem, menos rígida. Mais uma vez, surgem condições adversas ao desenvolvimento de modelagens confiáveis: existência de troncos jovens e galhos finos, em contraposição a outros galhos grossos e velhos; casos em que o cerne é muito menos rígido ou que ocupe mais de $30 \%$ do raio da madeira; ou nos casos de troncos ocos, por exemplo, em que a eficiência da estrutura pode chegar a ser três vezes mais estável que a estrutura de um tronco comum. Portanto, a modelagem nem sempre é um processo replicável, apesar da existência nos diversos modelos de um ponto em comum: os mecanismos de produção de madeira de reação nas diversas espécies são análogos, ou seja, existe um objetivo similar de aumentar a eficiência da estrutura a partir da indução do crescimento excêntrico mais espesso no lado da madeira de reação (ALMÉRAS; FOURNIER, 2009).

Em termos das caraterísticas das espécies e sua integração no ambiente, há que se mencionar o potencial natural que a árvore tem para apodrecer, ou seja, o que se espera em termos de decaimento da madeira, como processo natural por meio do qual a celulose e a lignina são convertidas em dióxido de carbono e água. O processo de decaimento pode chegar ao extremo de transformar a árvore em uma estrutura oca, ainda assim estável. A importância de se estudar as vantagens e desvantagens biofísicas de que as plantas tenham partes ocas, em seu ambiente natural, está na compreensão da ecologia das plantas e suas interações com o meio, enquanto que no ambiente urbano esta necessidade está em geral vinculada ao aumento do risco à vida e ao patrimônio (SPATZ; NIKLAS, 2013).

Tendo em conta as dificuldades inerentes à análise multivariada, há instituições de proteção das florestas e organismos internacionais, como a Sociedade Internacional de Arboricultura, que fomentam a criação de Guias de orientação para identificação e avaliação de árvores consideradas perigosas à segurança pública em termos de potencial para causar danos a pessoas e ao património público ou privado, bem como oferecem serviços de protocolos de análise de risco e credenciamento para controle de qualidade dos profissionais arborícolas. A intenção não é extinguir a probabilidade de ocorrência de danos provocados por 
árvores de risco, mas, sim, prover o julgamento profissional de uma abordagem sistemática que possibilite aos profissionais minimizar o erro de suas avaliações. Independentemente do tipo de método ou da técnica utilizada a análise de risco de queda deve idealmente compreender a inspeção interna e externa. Nesse processo diagnóstico não destrutivo de árvores em áreas urbanas é de grande auxílio para gestores e arboristas. As técnicas distintas e complementares entre si podem ser resumidas em: avaliação visual, testes acústicos, de resistência e análises bioquímicas (ALLISON; WANG, 2015).

O objetivo desta pesquisa é analisar o risco de queda de árvores, desenvolvendo e aplicando um formulário de avaliação da sanidade das árvores por meio de inspeção visual, bem como utilizando a tomografia de árvores como ferramenta de diagnóstico da avaliação da condição mecânica da estrutura do tronco. Espera-se contribuir para o fomento de mecanismos que contribuam para a adequada análise de risco de queda de árvores no ambiente urbano, auxiliando na gestão dos recursos públicos, seja pela prevenção de gastos desnecessários com o corte de árvores, seja pela possibilidade de monitorar ao longo do tempo a estrutura das árvores, propiciando a tomada de decisão de maneira tecnicamente fundamentada.

\section{MATERIAL E MÉTODOS}

A espécie selecionada foi Tilia tomentosa (Tilia), conhecida em inglês pelos nomes comuns Silver Linden, Basswood e Silver Lime. De acordo com o Sistema de Classificação Cronquist, a espécie pertence à família Tiliaceae. Entretanto, com o avanço dos estudos genéticos, a espécie passou a integrar a família Malvaceae, conforme classificação do Angiosperm Phylogeny Group - APG III, por similaridades das linhagens evolutivas das plantas (THE ANGIOSPERM PHYLOGENY GROUP, 2009).

A identificação da espécie foi conferida com as características do exemplar registado sob o no FFUC00000565, no Herbário da Universidade de Coimbra e de acordo com o processo de património público registado sob o no IPA 00010879, a espécie foi plantada no local de estudo na década de 20 do século passado.

Trata-se de árvore originariamente encontrada na Europa Oriental, nomeadamente na Península Balcânica, Hungria e Ucrânia, atualmente pode ser encontrada em quase toda a Península Ibérica, sendo, portanto, em Portugal uma árvore de origem alóctone capaz de atingir os $30 \mathrm{~m}$ de altura em condições ideais. As estimativas de quantidade de espécies dentro do género Tilia são bastante variáveis, e tendo em conta que todas as tílias podem ser propagadas por estaquia, enxerto e semente, a contabilização de híbridos também fica dificultada. A propagação por semente é notoriamente difícil, pois quando estas secam entram 
em dormência profunda e podem levar meses para germinar. Na Europa, há tílias que atingiram idades consideráveis de até dois mil anos (FUNDAÇÃO SERRALVES, 2016).

O local de estudo, Parque Dr. Manuel Braga, é conhecido como Parque da Cidade de Coimbra em Portugal. A área está situada na margem direita do rio Mondego e é um espaço verde que figura entre os atrativos turístico-ambientais, pela promoção de lazer, contemplação e desporto, considerado fundamental para a boa organização do espaço urbano e para a qualidade de vida dos cidadãos. Assim, a queda de ramos ou o colapso de um indivíduo arbóreo no Parque Dr. Manuel Braga pode acarretar danos ao património público, assim como comprometer a segurança dos transeuntes, visto ser a área verde em questão bastante frequentada, repleta de bancos debaixo das árvores, para além de ser área de acesso a outras partes das margens do rio Mondego. Posto isso, no que tange a análise de alvos, certamente toda a área, onde estão localizadas as tílias, é considerada de alto risco.

Após o reconhecimento da área e a realização de uma inspeção prévia do local, com o objetivo de melhor conhecer a condição das árvores a serem estudadas, constatou-se que: a) as árvores apresentam um fuste direito, com níveis leves de inclinação (Figura 1A); b) há danos, protuberâncias e fissuras em algumas das bases das árvores que podem facilitar a entrada de patogénicos, humidade e propiciar condições de comprometimento do cerne e, consequentemente, da estrutura da árvore (Figura 1B); c) há muitos ramos com condição aparente de poda e/ou com sinais de podridão, como buracos e fissuras longitudinais, em especial, quando foram observados os ramos centrais de algumas árvores (Figura 1C).

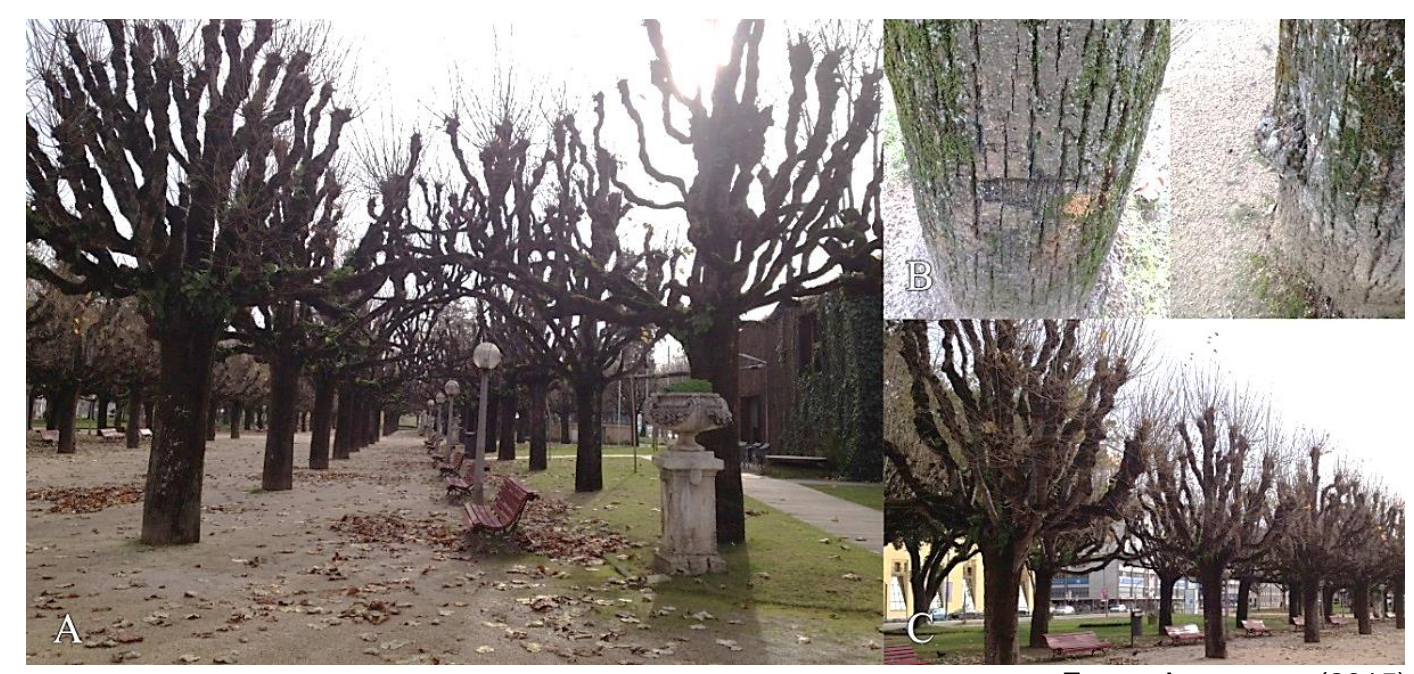

Fonte: As autoras (2015)

Figura 1. Tílias objeto de estudo. A. Árvores em alameda. B. Protuberâncias em condição irregular da base. C. Arquitetura antropizada dos ramos

Figure 1. Linden trees object of study. A. Straightness of mall trees. B. Bulges causing irregular base condition. C. Differentiated branch architecture from pruning 
Os sistemas de pontos existentes para avaliação do risco de queda de árvores no ambiente urbano, revisados pelo INSTITUTE OF FOOD AND AGRICULTURAL SCIENCES (2019) serviram de inspiração para a definição da metodologia utilizada para a realização da inspeção visual, que teve início com a atribuição de numeração das 103 Tílias identificadas em alameda, conforme Imagem do GoogleEarth, trabalhada com CorelDraw (Figura 2).

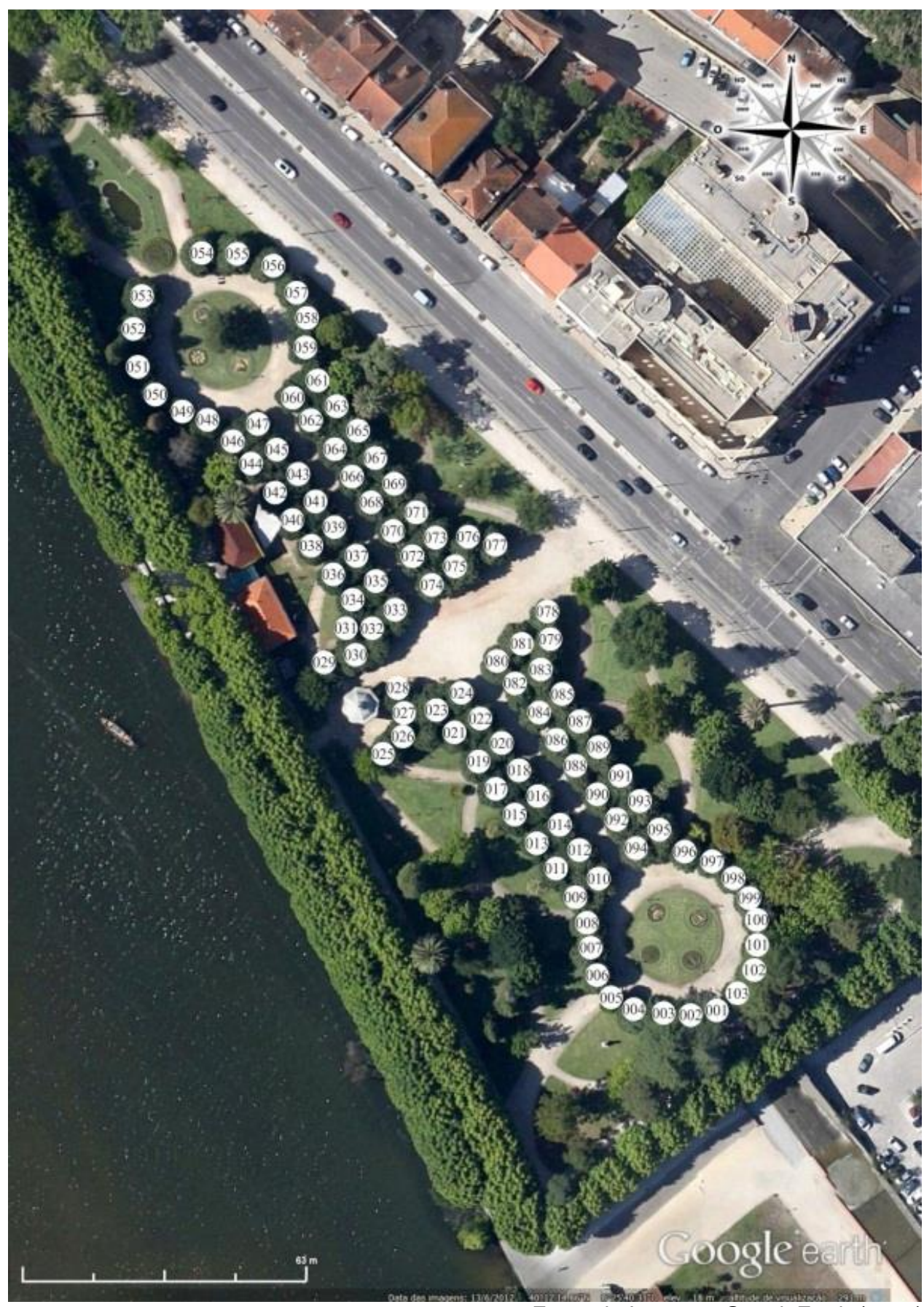

Fonte da Imagem: GoogleEarth (2012)

Figura 2. Imagem do local de estudo e numeração arbitrária das árvores para inspeção Figure 2. Study site image and arbitrary tree numbering for evaluation 
Levando em consideração os parâmetros potencialmente influenciadores da estabilidade mecânica, foram selecionados especificamente por representarem aspectos de discriminação visual entre os indivíduos, de acordo com a inspeção prévia realizada no local, ou seja, inclinação do tronco, condição irregular da base e ramos afetados por podas; bem como a existência de condições pouco usuais registradas em campo e que poderão vir a ser objeto de estudo, nomeadamente as interações por epifitismo e os ramos conectados em alameda, estabeleceu-se para cada parâmetro pontuação de 0 a 3, em categoria crescente, diretamente proporcional ao pior estado visual observado, conforme Formulário de inspeção visual das árvores, aplicado em dezembro de 2015 (Tabela 1).

Tabela 1. Formulário de inspeção visual das árvores utilizado no Parque Dr. Manuel Braga Table 1. Tree Visual Inspection Form used at Dr. Manuel Braga Park

\begin{tabular}{|c|c|c|}
\hline & & Pontuação por parâmetros e observações \\
\hline \multicolumn{3}{|l|}{ Parâmetros } \\
\hline \multirow{4}{*}{$\begin{array}{l}\text { Inclinação do Tronco - } \\
\text { TR }\end{array}$} & 0 & Ângulo zenital estimado $0^{\circ}$ ou nenhuma aparente inclinação \\
\hline & 1 & Ângulo zenital estimado até $5^{\circ}$ ou leve inclinação \\
\hline & 2 & Ângulo zenital estimado entre $5^{\circ}$ e $10^{\circ}$ ou direção de queda prevista \\
\hline & 3 & Ângulo zenital estimado entre $10^{\circ}$ e $15^{\circ}$ ou direção de queda nítida \\
\hline \multirow{4}{*}{$\begin{array}{l}\text { Condição } \\
\text { Irregular da } \\
\text { Base - CIB }\end{array}$} & 0 & Ausência de deformações na base do tronco \\
\hline & 1 & Alguma deformação na base do tronco \\
\hline & 2 & Mais de uma deformação na base do tronco \\
\hline & 3 & Muitas deformações na base do tronco \\
\hline \multirow{4}{*}{$\begin{array}{l}\text { Ramos afetados } \\
\text { por podas - RP }\end{array}$} & 0 & Nenhum ramo visualmente comprometido \\
\hline & 1 & Um ou poucos ramos visualmente comprometidos \\
\hline & 2 & Vários ramos visualmente comprometidos \\
\hline & 3 & Comprometimento de muitos ramos ou do ramo principal \\
\hline \multirow{4}{*}{$\begin{array}{l}\sum(\mathbf{T R}+\mathbf{C l B}+\mathbf{R P}) \\
\text { Estado } \\
\text { da Árvore - EA }\end{array}$} & 0 a 3 & \begin{tabular}{l|l} 
Verde & Bom estado \\
\end{tabular} \\
\hline & 4 & Amarelo $\mid$ Potencial de risco \\
\hline & 5 & Laranja | Potencial de risco elevado \\
\hline & 6 a 9 & Vermelho | Risco imediato \\
\hline \multicolumn{3}{|l|}{ Observações } \\
\hline \multirow{2}{*}{$\begin{array}{l}\text { Interações de } \\
\text { epifitismo - IB }\end{array}$} & 0 & Ausência visual de interação por epifitismo nos ramos \\
\hline & 1 & Presença visual de interação por epifitismo nos ramos \\
\hline \multirow{2}{*}{$\begin{array}{l}\text { Ramos conectados em } \\
\text { alameda - RC }\end{array}$} & 0 & Ausência de entrelaçamento de ramos de duas árvores distintas \\
\hline & 1 & Presença de entrelaçamento de ramos de duas árvores distintas \\
\hline
\end{tabular}

O somatório dos três parâmetros condicionou a classificação da árvore em valores de 0 a 9, compreendidos em classes de cores representativas do Estado fitossanitário segundo a inspeção visual. De ressaltar que para o enquadramento de classes por cores, não foram consideradas as pontuações de interações de epifitismo (IB) e ramos conectados em alameda 
$(\mathrm{RC})$, dada a dificuldade de estabelecer uma relação linear proporcional de seus efeitos sobre as estruturas das árvores. Espera-se que o diagnóstico das características de interações por epifitismo e o efeito de conexão dos ramos em alameda subsidiem estudos futuros das tílias.

A estratificação por cores resultante da classificação visual foi utilizada para selecionar aleatoriamente, 16 árvores para aplicação da tomografia de impulso na base, entre 15 e $30 \mathrm{~cm}$ do solo a depender do local imediato de inserção de raízes e no topo, entre 200 e $255 \mathrm{~cm}$, a depender do local imediato de inserção da copa em cada árvore, totalizando 32 tomografias. Optou-se por realizar uma tomografia na base e uma no topo de cada árvore selecionada, na tentativa de minimizar os erros relativos às inferências acerca da sanidade da árvore, já que os dados da tomografia são aplicáveis, apenas para a secção transversal analisada e pretende-se realizar a complementação da análise de risco da árvore, não de sua secção transversal.

O comportamento da velocidade da onda nos indivíduos arbóreos na população estudada pode ser razoavelmente homogêneo dentro de cada estrato, mas bastante heterogêneo em relação às velocidades das ondas sonoras esperadas para o total de indivíduos. A determinação das velocidades das ondas sonoras esperadas para a espécie Tilia tomentosa saudável poderá contribuir para o monitoramento da espécie em longo prazo, evitando que se precise intervir apenas quando a condição do espécime torna-se inaceitável em termos de risco no ambiente urbano. Assim, foram realizadas tomografias em indivíduos de cada grupo estratificado por cores; para assegurar que todos os estratos tenham representantes dentro da amostra, sob pena de ser ressaltada determinada característica do grupo, comprometendo a análise da variável de interesse que se encontra na população como um todo, qual seja o comportamento da velocidade da onda sonora para a espécie (NEGRINI et al., 2012).

O equipamento utilizado para realizar as tomografias foi 0 Arbotom, cujos componentes principais são uma bateria e os sensores, que são colocados no tronco na circunferência das árvores. Estes sensores propagam ondas sônicas pelo interior da árvore, a partir da batida de um martelo. A velocidade de propagação das ondas sônicas reflete o estado interno do lenho, gerando uma imagem no computador (Figura 3). 


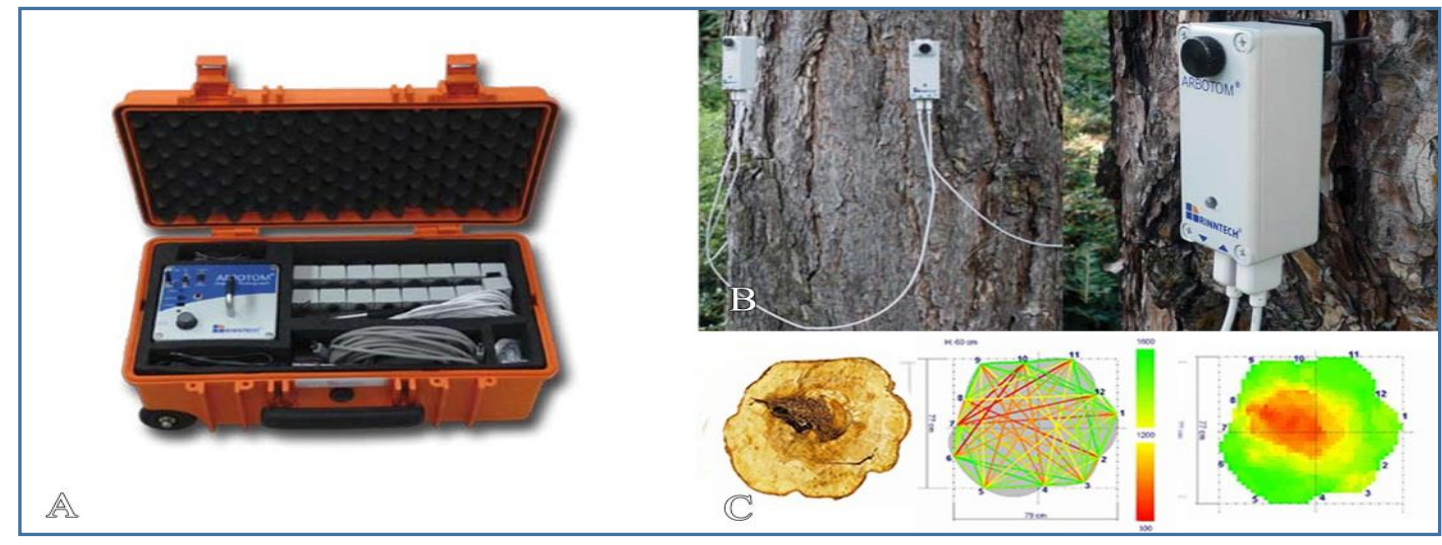

Fonte: RINNTECH, (2005)

Figura 3. Representação do equipamento Arbotom. A. Caixa. B. Sensores instalados. C. Resultados ilustrativos

Figure 3. Representation of the Arbotom equipment. The box. B. Sensors installed. C. Illustrative results

A altura da árvore foi medida com o auxílio de um clinômetro e a informação inserida nono programa que acompanha o equipamento utilizado. Utilizou-se uma bússola para determinar a direção norte onde foi sempre colocado o primeiro sensor. De seguida, foi dimensionada, com o auxílio de uma fita métrica, a circunferência da árvore à altura aproximada de $20 \mathrm{~cm}$ do solo, e posteriormente no topo à altura aproximada de $210 \mathrm{~cm}$ do solo, onde foram colocados os pregos a uma distância regular entre si, tanto quanto permitiam as reentrâncias da árvore e com uma força apenas suficiente capaz de suportar o peso dos sensores.

A distância entre cada sensor também foi mensurada a partir da referência do sensor localizado no sentido norte. Após a inserção dos dados, quando necessário, foram feitas correções das posições dos sensores, para indicar uma reentrância no sentido cerne-alburno ou negativos, nos casos de haver reentrâncias no sentido contrário. Cada sensor foi devidamente testado e foram realizadas entre cinco e oito batidas em cada sensor, para obtenção dos dados.

O programa do equipamento deve produzir três gráficos: o gráfico de linhas, o gráfico bidimensional e o gráfico mecânico. O gráfico bidimensional reflete as diferentes velocidades que o impulso decorrente da pancada física no sensor assume em face das condições da madeira. O gráfico de cores é formulado a partir do gráfico de linhas, por meio de um algoritmo matemático, que interpola as informações absolutas de velocidade, de modo a criar um valor na forma de área. Já para elaboração do gráfico mecânico, o software considera três tipos de informação acerca da árvore: o momento geométrico, baseado na forma da secção; o momento pesado, relativo ao decaimento interno; e o momento relativo de resistência, que indica a direção de queda em caso de falha da árvore. 


\section{RESULTADOS E DISCUSSÃO}

De acordo com os resultados obtidos após a Inspeção Visual por comparação entre os exemplares/árvores, verificou-se que os dois parâmetros que ressaltam as diferenças entre as árvores são: Inclinação do Tronco e Condição Irregular da Base, que são ramos afetados por podas de controle ao longo dos anos, tornaram muitos ramos ocos e quebradiços, em uma espécie de copa naturalmente arredondada, podas de topo comprometeram principalmente os ramos internos da copa.

Já a variável comum para a condição da árvore quanto à sanidade, foi a existência de muitos ramos com problema, ou de comprometimento do ramo principal, parâmetro frequente em 84/103 (Tabela 1). A realização de podas de controle ao longo dos anos tornaram muitos ramos ocos e quebradiços. Nesta espécie de copa naturalmente arredondada, podas de altura podem comprometer os ramos internos da copa, em especial aquele ramo que seria o principal da árvore, mas pelas intervenções humanas, tornaram-se um galho comum. O epifitismo é observável em 100/103 exemplares. Esse resultado pode orientar estudos da ecologia do género Tília.

Há 37 exemplares classificados com a cor verde, indicada para boa sanidade; 35 classificados com a cor amarela, com alerta para o estado de sanidade; 25 classificados com a cor laranja, indicando estado crítico; e seis com a cor vermelha, estado altamente crítico (Tabela 1).

Tabela 1. Análise Visual do estado de conservação das tílias do Parque Manuel Braga, Coimbra Table 1. Visual analysis in terms of risk of the linden trees of the Manuel Braga Park, Coimbra

\begin{tabular}{cccccccc|cccccccc}
\hline \multicolumn{3}{c}{ Parâmetros } & \multicolumn{3}{c}{ Classificação } & \multicolumn{3}{c}{ Obs. } & \multicolumn{3}{c}{ Parâmetros } & \multicolumn{3}{c}{ Classificação } & \multicolumn{2}{c}{ Obs. } \\
\hline ID & TR & CIB & RP & $\Sigma$ & EA & RC & IB & ID & TR & CIB & RP & $\Sigma$ & EA & RC & IB \\
\hline $\mathbf{1}$ & 2 & 1 & 3 & 6 & & 0 & 0 & $\mathbf{5 2}$ & 0 & 1 & 0 & 1 & & 0 & 1 \\
$\mathbf{2}$ & 2 & 0 & 2 & 4 & & 0 & 0 & $\mathbf{5 3}$ & 2 & 1 & 0 & 3 & & 0 & 1 \\
$\mathbf{3}$ & 1 & 2 & 2 & 5 & & 0 & 0 & $\mathbf{5 4}$ & 0 & 0 & 2 & 2 & & 0 & 1 \\
$\mathbf{4}$ & 3 & 0 & 2 & 5 & & 0 & 1 & $\mathbf{5 5}$ & 1 & 0 & 2 & 3 & & 0 & 1 \\
$\mathbf{5}$ & 1 & 1 & 1 & 3 & & 0 & 1 & $\mathbf{5 6}$ & 1 & 0 & 1 & 2 & & 0 & 1 \\
$\mathbf{6}$ & 1 & 0 & 2 & 3 & & 0 & 1 & $\mathbf{5 7}$ & 0 & 1 & 2 & 3 & & 0 & 1 \\
$\mathbf{7}$ & 0 & 2 & 3 & 5 & & 1 & 1 & $\mathbf{5 8}$ & 0 & 1 & 2 & 3 & & 0 & 1 \\
$\mathbf{8}$ & 0 & 0 & 2 & 2 & & 1 & 1 & $\mathbf{5 9}$ & 0 & 0 & 1 & 1 & & 0 & 1 \\
$\mathbf{9}$ & 0 & 2 & 3 & 5 & & 1 & 1 & $\mathbf{6 0}$ & 1 & 1 & 2 & 4 & & 0 & 1 \\
$\mathbf{1 0}$ & 2 & 0 & 2 & 4 & & 1 & 1 & $\mathbf{6 1}$ & 0 & 1 & 3 & 4 & & 0 & 1 \\
$\mathbf{1 1}$ & 1 & 1 & 2 & 4 & & 1 & 1 & $\mathbf{6 2}$ & 2 & 0 & 1 & 3 & & 1 & 1 \\
$\mathbf{1 2}$ & 1 & 0 & 3 & 4 & & 1 & 1 & $\mathbf{6 3}$ & 0 & 0 & 3 & 3 & & 1 & 1 \\
$\mathbf{1 3}$ & 2 & 1 & 2 & 5 & & 1 & 1 & $\mathbf{6 4}$ & 1 & 1 & 3 & 5 & & 1 & 1 \\
$\mathbf{1 4}$ & 2 & 1 & 2 & 5 & & 1 & 1 & $\mathbf{6 5}$ & 0 & 0 & 3 & 3 & & 1 & 1 \\
$\mathbf{1 5}$ & 2 & 0 & 2 & 4 & & 1 & 1 & $\mathbf{6 6}$ & 1 & 1 & 1 & 3 & & 0 & 1 \\
$\mathbf{1 6}$ & 1 & 1 & 3 & 5 & & 1 & 1 & $\mathbf{6 7}$ & 1 & 1 & 1 & 3 & & 0 & 1 \\
$\mathbf{1 7}$ & 1 & 0 & 3 & 4 & & 0 & 1 & $\mathbf{6 8}$ & 2 & 0 & 1 & 3 & & 0 & 1 \\
$\mathbf{1 8}$ & 1 & 0 & 3 & 4 & & 0 & 1 & $\mathbf{6 9}$ & 1 & 1 & 1 & 3 & & 0 & 1
\end{tabular}




\begin{tabular}{|c|c|c|c|c|c|c|c|c|c|c|c|c|c|c|c|}
\hline \multirow[b]{2}{*}{ ID } & \multicolumn{3}{|c|}{ Parâmetros } & \multicolumn{2}{|c|}{ Classificação } & \multicolumn{3}{|c|}{ Obs. } & \multicolumn{3}{|c|}{ Parâmetros } & \multicolumn{2}{|c|}{ Classificação } & \multicolumn{2}{|c|}{ Obs. } \\
\hline & TR & CIB & $\mathbf{R P}$ & $\Sigma$ & EA & RC & IB & ID & TR & CIB & $\mathbf{R P}$ & $\Sigma$ & EA & RC & IB \\
\hline 19 & 1 & 0 & 2 & 3 & & 0 & 1 & 70 & 0 & 0 & 3 & 3 & & 1 & 1 \\
\hline 20 & 2 & 0 & 2 & 4 & & 0 & 1 & 71 & 1 & 2 & 3 & 6 & & 1 & 1 \\
\hline 21 & 1 & 1 & 3 & 5 & & 0 & 1 & 72 & 1 & 1 & 3 & 5 & & 1 & 1 \\
\hline 22 & 2 & 0 & 2 & 4 & & 0 & 1 & 73 & 2 & 0 & 1 & 3 & & 1 & 1 \\
\hline 23 & 2 & 0 & 2 & 4 & & 1 & 1 & 74 & 2 & 1 & 2 & 5 & & 1 & 1 \\
\hline 24 & 1 & 1 & 2 & 4 & & 1 & 1 & 75 & 1 & 2 & 3 & 6 & & 1 & 1 \\
\hline 25 & 1 & 1 & 2 & 4 & & 0 & 1 & 76 & 0 & 1 & 3 & 4 & & 0 & 1 \\
\hline 26 & 1 & 0 & 3 & 4 & & 0 & 1 & 77 & 2 & 0 & 3 & 5 & & 0 & 1 \\
\hline 27 & 1 & 0 & 3 & 4 & & 0 & 1 & 78 & 2 & 1 & 3 & 6 & & 0 & 1 \\
\hline 28 & 1 & 1 & 3 & 5 & & 0 & 1 & 79 & 2 & 2 & 3 & 7 & & 0 & 1 \\
\hline 29 & 1 & 1 & 3 & 5 & & 0 & 1 & 80 & 1 & 1 & 2 & 4 & & 0 & 1 \\
\hline 30 & 2 & 0 & 2 & 4 & & 0 & 1 & 81 & 1 & 1 & 2 & 4 & & 0 & 1 \\
\hline 31 & 1 & 1 & 2 & 4 & & 0 & 1 & 82 & 1 & 2 & 3 & 6 & & 1 & 1 \\
\hline 32 & 1 & 2 & 2 & 5 & & 1 & 1 & 83 & 2 & 0 & 2 & 4 & & 1 & 1 \\
\hline 33 & 0 & 1 & 3 & 4 & & 1 & 1 & 84 & 1 & 1 & 3 & 5 & & 1 & 1 \\
\hline 34 & 1 & 1 & 2 & 4 & & 1 & 1 & 85 & 0 & 2 & 3 & 5 & & 1 & 1 \\
\hline 35 & 0 & 2 & 3 & 5 & & 1 & 1 & 86 & 0 & 2 & 2 & 4 & & 0 & 1 \\
\hline 36 & 1 & 2 & 1 & 4 & & 1 & 1 & 87 & 1 & 0 & 3 & 4 & & 0 & 1 \\
\hline 37 & 1 & 1 & 1 & 3 & & 1 & 1 & 88 & 0 & 2 & 3 & 5 & & 0 & 1 \\
\hline 38 & 0 & 1 & 2 & 3 & & 0 & 1 & 89 & 1 & 0 & 3 & 4 & & 0 & 1 \\
\hline 39 & 0 & 0 & 3 & 3 & & 1 & 1 & 90 & 0 & 2 & 3 & 5 & & 0 & 1 \\
\hline 40 & 1 & 0 & 2 & 3 & & 0 & 1 & 91 & 1 & 1 & 3 & 5 & & 0 & 1 \\
\hline 41 & 1 & 0 & 2 & 3 & & 0 & 1 & 92 & 1 & 3 & 3 & 7 & & 0 & 1 \\
\hline 42 & 0 & 1 & 2 & 3 & & 0 & 1 & 93 & 2 & 0 & 2 & 4 & & 0 & 1 \\
\hline 43 & 1 & 0 & 2 & 3 & & 0 & 1 & 94 & 0 & 1 & 2 & 3 & & 1 & 1 \\
\hline 44 & 0 & 0 & 2 & 2 & & 0 & 1 & 95 & 0 & 1 & 3 & 4 & & 1 & 1 \\
\hline 45 & 1 & 1 & 3 & 5 & & 1 & 1 & 96 & 1 & 1 & 3 & 5 & & 0 & 1 \\
\hline 46 & 0 & 0 & 1 & 1 & & 0 & 1 & 97 & 1 & 0 & 2 & 3 & & 0 & 1 \\
\hline 47 & 1 & 1 & 1 & 3 & & 0 & 1 & 98 & 0 & 1 & 3 & 4 & & 0 & 1 \\
\hline 48 & 1 & 0 & 2 & 3 & & 0 & 1 & 99 & 0 & 2 & 2 & 4 & & 0 & 1 \\
\hline 49 & 1 & 3 & 2 & 6 & & 0 & 1 & 100 & 1 & 1 & 2 & 4 & & 0 & 1 \\
\hline 50 & 1 & 1 & 1 & 3 & & 0 & 1 & 101 & 1 & 0 & 2 & 3 & & 0 & 1 \\
\hline \multirow[t]{2}{*}{51} & 0 & 1 & 0 & 1 & & 0 & 1 & 102 & 1 & 0 & 2 & 3 & & 0 & 1 \\
\hline & & & & & & & & 103 & 0 & 1 & 2 & 3 & & 0 & 1 \\
\hline
\end{tabular}

Como possível causa das diferenças observadas entre árvores, no que tange ao seu estado de sanidade, apontamos a possibilidade de que o comportamento do lençol freático se tenha alterado, influenciando a disponibilização de água e nutrientes para as raízes de maneira desigual, por ser mais rebaixado em algumas áreas do parque. Isto porque, as águas subterrâneas alcançam o leito do rio de maneira não uniforme. Toda a área do Parque é oriunda de um grande aterro, criado para contenção das cheias do rio Mondego, e, portanto, trata-se de solo que se encontra alterado abaixo do nível das raízes das árvores em até, no mínimo, cinco metros, que é o equivalente à altura de compactação do solo anteriormente ao plantio das árvores. Neste caso, é possível que as raízes de algumas árvores tenham menos 
ou mais facilidade de acesso à água em períodos de estiagem, sendo, portanto, mais afetadas relativamente aos demais exemplares.

Quanto aos resultados das tomografias acústicas realizadas, o gráfico mecânico pode ser considerado o mais adequado para análise da sanidade da árvore, pelo fato de o gráfico mecânico refletir de forma mais clara e sucinta os resultados parciais, ou seja, os gráficos de linhas, os gráficos de densidades, bem como as tabelas de velocidade da onda sonora, que são inerentes a cada tomografia. Resultados potencialmente preocupantes quanto ao risco de queda são encontrados quando o valor do momento geométrico apresenta-se abaixo de $50 \%$ (RINNTECH, 2005).

Tal comportamento pode ser observado na tomografia do exemplar 01, classificado como de estado de sanidade crítico. O exemplar apresenta um tronco circular na base e na porção superior do tronco, sendo ambas as secções transversais relativamente círculos perfeitos, com estabilidade de 99-100\%. A característica de elevada simetria, quase perfeição cilíndrica, do tronco dos exemplares estudados é destacável e foi relevante para o alto valor de estabilidade, mesmo para este espécimen. Quando condições, naturais ou não, relativas a reentrâncias, irregularidades ou comportamento ovalar na secção do tronco, se manifestam, a estabilidade percentual acaba por ser afetada negativamente.

Quanto ao decaimento ou possível fragilidade da árvore, tanto na secção da base, quanto na secção do topo, há manchas ao nível do rosa, que indicam perda substancial de suporte físico para as ondas emitidas pelo equipamento.

Para ambas as secções, a variação observada parece influenciar de forma similar a resistência à flexão, de $72-87 \%$ para a base e de $73-87 \%$ para o topo. As condições das secções transversais circulares da base e do topo, quanto à forma, são similares e quanto ao decaimento da madeira, igualmente em mau estado e com indicações de possível direção de queda bastante semelhante: Sudoeste (SO). Da razão entre a componente geométrica e a componente mecânica, obtém-se que a resistência à flexão da árvore é de 73-87\% (Figura 4).

Argumentamos que as diferenças entre imagens em que constam as cores verdes e amarelas são praticamente imperceptíveis quando analisados apenas os dados de velocidade média. Isto porque, o intervalo de velocidades médias das imagens em que aparecem as colorações verdes | amarelas | laranjas | vermelhos são respetivamente: $941 \rightarrow 1056$ | $818 \rightarrow 1137|736 \rightarrow 880| 477 \rightarrow 506 \mathrm{~m} / \mathrm{s}$. Depreende-se que um mesmo valor de velocidade pode ser enquadrado pelo tomógrafo como cor verde ou amarela e que quando a cor vermelha é apresentada na tomografia é porque de fato não há dúvidas quanto ao comprometimento da estrutura, em relação à sua resposta diante da onda sonora. 

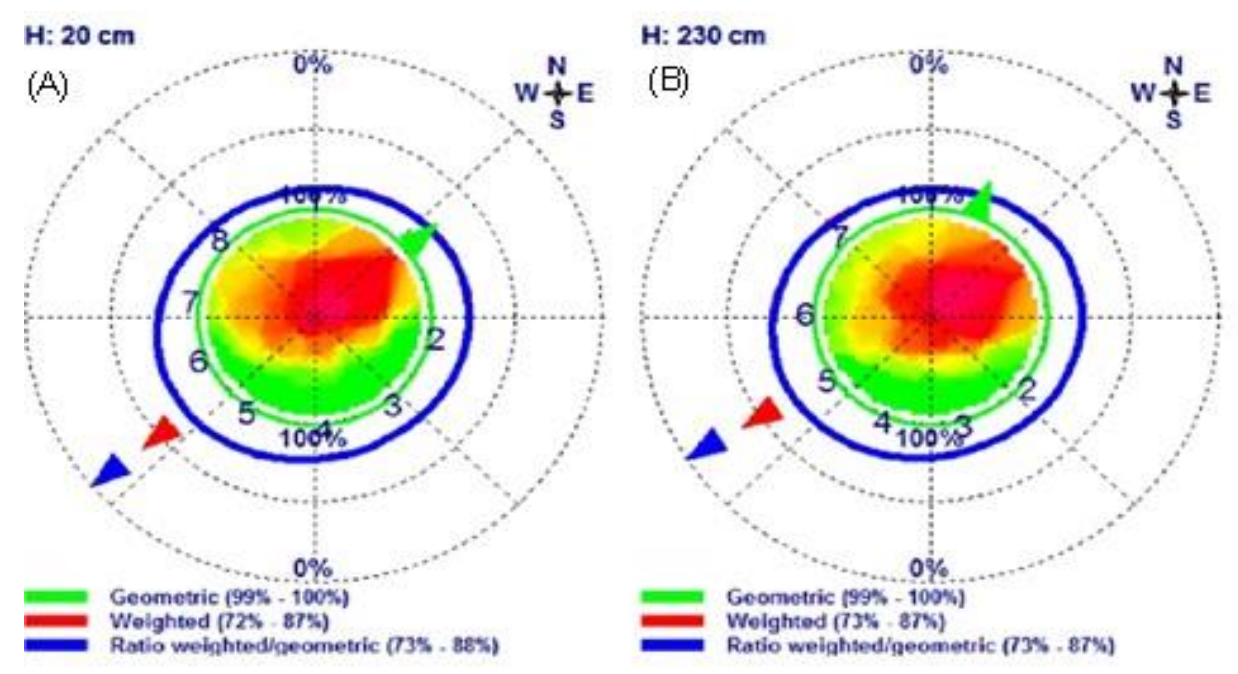

Figura 4. Tomografia do Exemplar 01. Gráficos mecânicos da base (A) e do topo (B)

Figure 4. Exemplary Tomography 01. Mechanical charts of the base and top

O tomógrafo, como equipamento que recolhe dados representativos da condição interna da árvore, permite uma boa aproximação acerca da sanidade desta. No entanto, por si só não é suficiente para atestar eventual condição de risco, fazendo-se necessárias outras componentes para uma avaliação completa. Em relação à amostra de 16 exemplares em que se utilizou o equipamento, para 14 dos 16 exemplares avaliados, a análise visual levou a resultados equivalentes ou mais rigorosos. Em apenas 2 casos o equipamento demonstrou haver uma condição interna pior, na base ou no topo, do que aquilo que foi observado externamente, nomeadamente para os exemplares 19 e 97.

A importância do uso do equipamento de tomografia fica evidenciada exatamente nos casos dos exemplares 19 e 97 . Isto porque, os casos mais preocupantes, em termos de risco mal avaliado, residem nas omissões ou falhas da análise humana, ou seja, quando nas análises visuais não são perceptíveis falhas internas que podem até mesmo levar à queda da árvore.

Em se tratando da componente Biodiversidade, a realização de tomografias em árvores poderia ser mais bem aproveitada, no sentido de também se utilizarem as diversas matrizes de dados fornecidas como resultados após a realização da tomografia acústica, para construir uma base de dados com intervalos de velocidade da onda de tensão na madeira que refletissem o padrão normal da espécie, ou seja, de árvores sadias.

Essas referências poderiam facilitar análises de risco de queda de árvores para portadores de equipamentos acústicos mais simples, do tipo sensor-receptor, que passariam a ter dados de referência para árvores saudáveis para a espécie Tilia tomentosa, e por comparação seria possível fazer inferências acerca da sanidade da árvore, se utilizando de 
menos sensores. Para, além disso, isolar o intervalo de madeira sã da espécie poderia vir a contribuir para estudos de diferenciação das espécies dentro do Gênero.

\section{CONCLUSÕES}

Em análise de risco de queda de árvores, há uma rotina básica de boas práticas que consiste em primeiramente avaliar a relevância dos alvos na zona de impacto no caso de colapso de pernadas ou mesmo de queda da árvore e posteriormente proceder à avaliação da árvore para que a tomada de decisão seja complementada pela probabilidade de falha mecânica, sempre que possível com o auxílio de equipamento próprio capaz de conferir objetividade à avaliação.

Com o uso do equipamento Arbotom, para uma amostra de 16 exemplares, a média de velocidade da propagação da onda das árvores consideradas saudáveis foi de $965 \mathrm{~m} / \mathrm{s}$, ou seja, apenas o exemplar 01 da amostra apresentou valores médios de velocidade inferiores a $724 \mathrm{~m} / \mathrm{s}$. Mesmo os exemplares considerados comprometidos na análise visual, quando submetidos à inspeção por equipamento, provaram ser capazes de manter a estabilidade mecânica de sua estrutura. O corte de uma árvore pode comprometer indiretamente a disponibilidade de recursos para fungos e micro-organismos e, consequentemente, reduzir os níveis de Biodiversidade no ambiente urbano.

Recomenda-se que as Inspeções visuais sejam acompanhadas pela avaliação com o uso de um equipamento de diagnóstico não destrutivo, no sentido de serem prevenidas conclusões equivocadas acerca do risco de queda de uma árvore. Portanto, idealmente, devese aplicar a análise múltipla como forma de minimização dos riscos.

É desejável desenvolver um banco de dados com as velocidades por espécie e realizar novas medições com frequência anual nos mesmos exemplares da amostra, a fim de monitorar o estado de decaimento natural do lenho ao longo dos anos.

\section{AGRADECIMENTOS}

Aos colegas da Universidade de Coimbra e à Maria João Martins, da Câmara Municipal de Coimbra, por terem viabilizado a realização deste trabalho. 


\section{REFERÊNCIAS}

ALLISON, R. B., WANG, X. Nondestructive testing in the Urban Forest. In: ROSS, J. R. (Ed.). Nondestructive evaluation of wood. Wisconsin: Forest Products Laboratory/United States Department of Agriculture, 2015. p. 82-93.

ALMÉRAS, T., FOURNIER, M. Biomechanical design and long-term stability of trees: Morphological and wood traits involved in the balance between weight increase and the gravitropic reaction. Journal of Theoretical Biology, France, v. 256, n. 3, p. 370-381, 2009.

BUCKERIDGE, M. Árvores urbanas em São Paulo: planejamento, economia e água. Estudos avançados, São Paulo, v. 29, n. 84, p. 85-101, 2015.

FERREIRA, A. J. D.; PARDAL, J.; MALTA, M.; FERREIRA, C. S. S. , SOARES, D. D. J.; VILHENA, J. Improving Urban Ecosystems Resilience at a City Level the Coimbra Case Study. Energy Procedia, Coimbra/Portugal, v. 40, p. 6-14, 2013.

FUNDAÇÃO SERRALVES. Acervo de dados de Tilia tomentosa. Disponível em:<http://biodiversidade.serralves.pt/pt/> Acesso em: 26 out. 2016.

\section{INSTITUTE OF FOOD AND AGRICULTURAL SCIENCES. Tree Risk Assessment Methods:} A Comparison of Three Common Evaluation Forms. Disponível em:<https://edis.ifas.ufl.edu/ep487> Acesso em 09 set. 2019.

NEGRINI, M.; AGUIAR, M. D. DE; VIEIRA, C. T.; SILVA, A. C. DA; HIGUCHI, P. Dispersão, distribuição espacial e estratificação vertical da comunidade arbórea em um fragmento florestal no Planalto Catarinense. Revista Árvore, v. 36, n. 5, p. 919-930, 2012.

PAIVA, J. As artérias urbanas e as árvores. Anais do I Colóquio de Geografia, Coimbra, Cadernos de Geografia, n. especial, p. 49-55, 1996.

RINNTECH. Three-dimensional Impulse Tomograph for Examination of Trees and Timber. Arbotom Manual, Germany, p. 42, 2005.

RUXTON, G. D. Why are so many trees hollow? Biology Letters, London, v. 10, p. 1-3, 2014.

SHORTLE, W. C., DUDZIK, K. R., \& SMITH, K. T. Development of wood decay in woundinitiated discolored wood of eastern red cedar. Holzforschung - International Journal of the Biology, Chemistry, Physics and Technology of Wood, v. 64, n. 4, p. 529-536, 2010.

SPATZ, H. C.; NIKLAS, K. J. Modes of failure in tubular plant organs. American Journal of Botany, v. 100, n. 2, p. 1-7, 2013.

THE ANGIOSPERM PHYLOGENY GROUP. An update of the Angiosperm Phylogeny Group classification for the orders and families of flowering plants: APG III. Botanical Journal of the Linnean Society, London, n.161, p. 105-121, 2009. 